\title{
DISCUSSION
}

\section{Drained principal stress rotation in saturated sand}

\author{
M. J. SYMES, A. GENS and D. W. HIGHT (1988). Géotechnique 38, No. 1, 59-81
}

\section{A. S. F. Sayo and Y. P. Vaid, University of British Columbia}

In this and a series of earlier papers, the Authors have made important contributions to the subject of principal stress rotation effects on soil behaviour. In particular, their interpretation of results from a hollow cylinder torsional test utilizing the concept of a bounding surface (BS) provides a simple framework for a qualitative understanding of the effects of principal stress rotation on sand behaviour. Some aspects of data interpretation may have an important bearing on the Authors' specimen preparation procedure. We present experimental evidence that shows the BS may not remain symmetrical about $\alpha=0$ plane after the sand has been subjected to principal stress rotation on either side of the vertical and caution against the conclusion that the strain response along a given stress path is independent of the prior stress history, provided current stress state lies on the BS.

\section{Specimen preparation procedure}

Before the start of every HCT test reported by the Authors, a drained triaxial compression cycle is routinely applied to the specimen. Loose specimens are preloaded to a principal stress ratio $\left(R=\sigma_{1}{ }^{\prime} / \sigma_{3}{ }^{\prime}\right)$ equal to 2.0 and dense ones to $R=2 \cdot 6$ (Symes, 1983). These $R$ levels were shown to represent more than $50 \%$ of the maximum shear strength. It is not clear whether these socalled triaxial compression cycles are applied with $p^{\prime}$ held constant at $200 \mathrm{kPa}$ or just by increasing the vertical load with $\sigma_{\mathrm{r}}{ }^{\prime}$ held constant at $200 \mathrm{kPa}$. In the latter case, $p^{\prime}$ is also simultaneously cycled between 200 and $267 \mathrm{kP}$ ) (loose specimens) or between 200 and $307 \mathrm{kPa}$ (dense specimens). This load-unload preparation cycle is likely to have significant hardening effects on the strain response of sand specimens on subsequent testing phases, in the region of $R$ and $p^{\prime}$ values below the maximum preload values.

For the stress conditions reported by the Authors $\left(p^{\prime}=200 \mathrm{kPa}, q=43 \mathrm{kPa}, b=0.5\right.$ and $\alpha=0$ or $45^{\circ}$ ), principal stress rotations in tests LR1 and LR2 were applied at $R=1.55$, that is well below the maximum perload value of $2 \cdot 0$. As a consequence, the normalized BS, presented in Figs 11 and 12, has most of its pre-peak region not corresponding to primary loading conditions. Thus, normal and shear strains induced during principal stress rotation in test LR1 (path BF, Fig. 12) should be expected to be relatively small. This indeed is the case as seen in Figs 6 and 7. Strains are much smaller in test LR 1 than in test LR3 in which rotation takes place at $R$ greater than the preload values of $2 \cdot 0$. Further evidence in support of hardening effects in test LR1 is provided by the relative directions of major principal strain and stress increments shown in Fig. 16. In test LR $1, \alpha_{\varepsilon}$ is very close to $\alpha_{\sigma}$, suggesting that the stress path BF may be inside the BS and hence recoverable strains predominate. In test LR3, in contrast, $\alpha_{\varepsilon} \cdot$ lies much closer to $\alpha$ (major principal stress direction), indicating predominance of irrecoverable strains. This will be normally associated with primary loading conditions (stress paths traversing the BS). Thus, the differences in strain development during rotations in tests LR1 and LR3 are not only due to different $R$ values, as indicated by the Authors, but also due to the hardening effects of the preparation compression cycle on specimen LR1.

\section{Repositioning or reshaping of BS}

Symes et al. (1985) showed that the BS is symmetrical about the $\alpha=0$ plane. Also, in the present Paper, it is concluded that the strains developed during forward or backward principal stress rotation tests played little part in repositioning or reshaping the BS.

In accordance with these indications, the specimen's stress state after one full rotation cycle should lie beneath the BS. If the symmetrical shape or position of the BS has been unaffected, imposition of either a positive or negative second rotation cycle would result in identical strain 

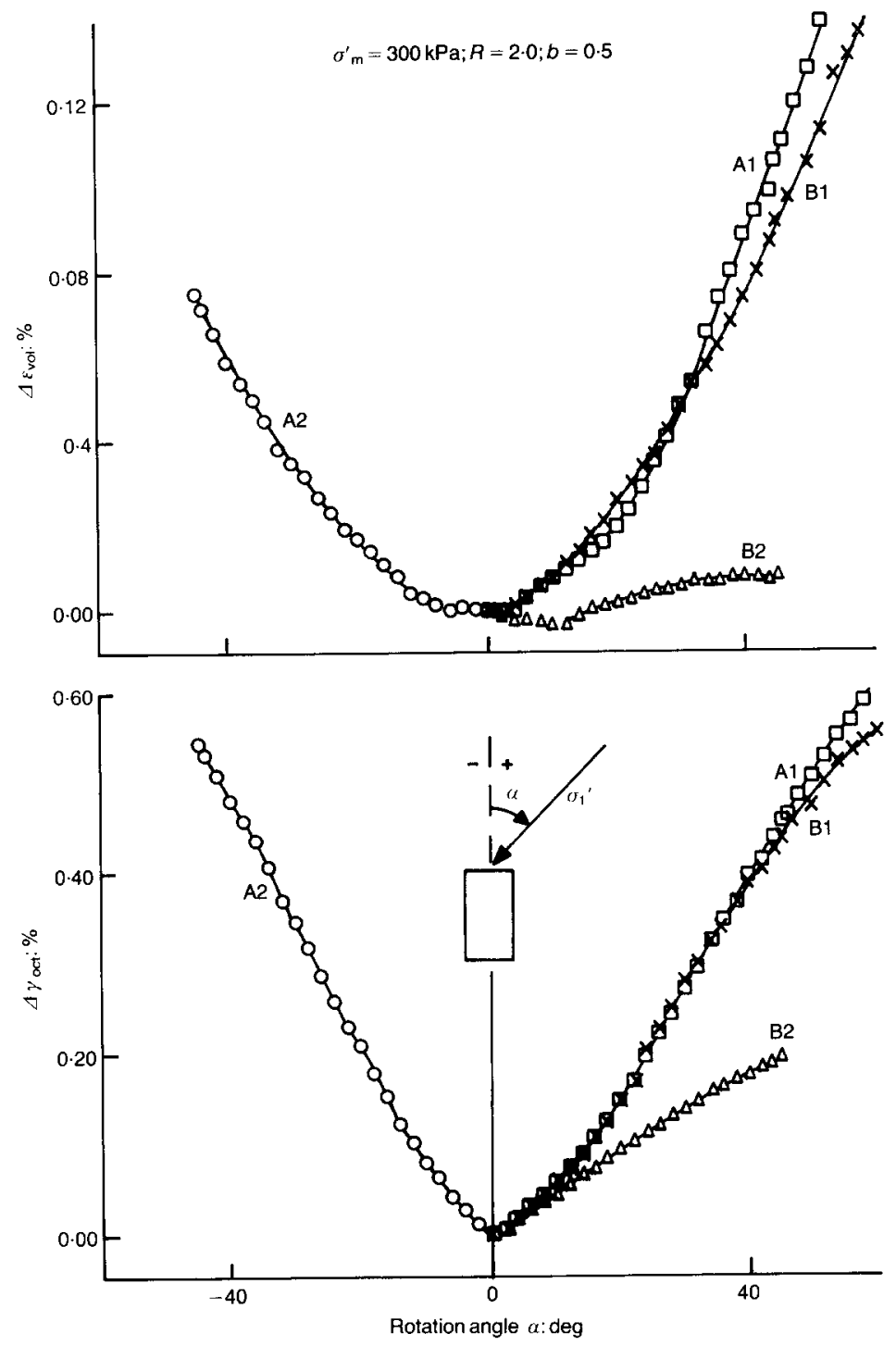

Fig. 34. Strain development during forward principal stress rotation phases

developments. This identity is not observed as shown by the results of HCT tests (Fig. 34) on a medium-loose, uniform, subrounded, quartz sand (Sayao, 1988). Two identical water pluviated specimens A and B $(h=30.5 \mathrm{~cm}, 0 . d .=15.2 \mathrm{~cm}$ and i.d. $=10 \cdot 2 \mathrm{~cm}$ ) were loaded to $R=2 \cdot 0$, $p^{\prime}=300 \mathrm{kPa}$ and $b=0.5$, with $\alpha=0$. First rotation cycles (A1 and B1) were then imposed to both specimens, in the positive $\alpha$ direction. The volumetric and shear strain developed during the forward rotation phase $\left(\alpha=0 \rightarrow+60^{\circ}\right)$ are shown in Fig. 34. The results show excellent repeatability. After reducing $\alpha$ back to zero, specimen $B$ was subjected to a second cycle of rotation in the same positive $\alpha$ direction, but specimen $A$ was subjected to rotation cycle in the opposite negative $\alpha$ direction. The strains developed during the second rotation phase are highly direction dependent. The strains are much smaller when reloading in the positive $\alpha$ direction than in the negative $\alpha$ direction. The strains developed during the negative rotation $A 2$ are similar to those developed during primary rotation (A1 or B1). It appears that the hardening effects observed 
during reloading in the same $\alpha$ direction is not remembered by the soil if rotation is imposed in the opposite direction. These results strongly suggest that in this case the position and/or shape of the BS should have been greatly affected by the first principal stress rotation cycle.

\section{Effect of previous stress history}

In interpreting their HCT test results, the Authors concluded that not only the shear strength but also the strain response along a given stress path are independent of the previous stress history, provided the current stress state is lying on the BS. However, directly opposite conclusions were arrived at in a previously reported HCT study on dense specimens at $R=3.5$ (Hight et al., 1983; Fig. 34). This apparent contradiction confirms that the concept of a BS should not be widely applied to all combinations of $R$ and $D_{\mathrm{r}}$, as suggested by the Authors.

\section{Authors' reply}

The first point of the discussion refers to the possible effects on the subsequent results of a preloading cycle applied to the specimen. The preloading cycle involved drained compression, with $\sigma_{2}$ equal to $\sigma_{3}$ and $p^{\prime}$ constant at $200 \mathrm{kPa}$; the stress ratio, $R\left(=\sigma_{1}{ }^{\prime} / \sigma_{3}{ }^{\prime}\right)$, was increased to $2 \cdot 0$ for loose specimens and 2.6 for dense specimens, before unloading along the same stress path. The reasons for performing this stress cycle were twofold: firstly to check the repeatability of the sample preparation procedure and secondly to apply to the specimen a stage of non-isotropic loading that would create a fabric in the material which should be, in principle, closer to that in the field, where purely isotropic consolidation is not likely to occur. Therefore, the tests were carried out on a material whose initial state was a consequence of both the sample preparation method and the previous stress history. In this context, the Authors agree that the strain response of the sand, and, in particular, the differences between the results of tests LR1 and LR3 were influenced by the preloading cycle applied to all the specimens. However, a similar overall pattern of behaviour to that reported in this and earlier papers has been observed in tests series where only isotropic consolidation, i.e. without any preloading cycle, had been applied to the specimens (Shibuya, 1985).

The remainder of the discussion refers to the conceptual model used in the Paper for the inter- pretaton of the results. The aim of the Authors when proposing this approach was to provide a unifying framework which took into account the new parameter of principal stress rotation. To this end, the framework was based on a concept, the bounding surface (BS), which, although not without drawbacks, has a long tradition of usefulness in soil mechanics. As shown in this Paper and in earlier papers (Symes et al. 1984; 1985) the framework is able to explain, in a consistent way, major features of behaviour such as

(a) the effect of principal stress rotation on failure conditions

(b) differences in volumetric behaviour, depending on the position of the state of the specimen with respect to the BS

(c) strain response differences, depending on the direction in which the principal stresses are rotated

(d) conditions for which liquefaction of a specimen subject to cyclic rotation of principal stress occurs

(e) the relationship between drained and undrained behaviour.

Therefore, knowledge of the shape and position of the BS for a particular state of the sand can be extremely valuable for predicting its future behaviour. However, as pointed out in the conclusions of the Paper, the BS is unlikely to remain unchanged throughout the entire stress history of the specimen, especially when large strains develop. The determination of the BS and its variation can, therefore, be considered as a promising approach for integrating the effects of principal stress rotations into a generalized framework for soil behaviour. In this respect, it is pleasing that the discussers analyse their results in terms of a BS. They show that, following a rotation from $\alpha=0^{\circ}$ to $\alpha=60^{\circ}$, the BS does not develop symmetrically around the $\alpha=0^{\circ}$ axis but it depends on the direction in which the first rotation was applied. This is an important result concerning a region of stress space not covered by the testing programme reported in the Paper and the Authors suspect that other types of stress reversals (defined in a generalized way including principal stress rotations) will have similar effects. The Authors wish to point out that in Symes et al. (1985), it was the initial BS that was shown to be symmetrical about the $\alpha=0^{\circ}$ axis; the subsequent evolution of the BS was not investigated.

Concerning the final point of the discussion, which refers to some preliminary tests carried out on dense sand, the Authors would like to emphasize that the predictions based on the BS concept should be expected to apply only to the major features of behaviour. In the Authors' opinion, the differences in behaviour reported in the 
Figure referred to in the Discussion are certainly not large enough to reach the conclusion that different stress paths result in qualitatively different behaviour, especially if it is considered that the results refer to strains close to failure (where nonuniformities of the specimen may become significant) and that the stress axis is in terms of stress ratio, a parameter that varies rapidly when approaching failure. It remains the Authors' view, based on observations in several test series in the HCA, that the strain response for a stress path traversing the BS is largely independent of the path taken to reach the current stress state on the BS.

\section{REFERENCES}

Sayao, A. S. F. (1988). General stress path testing of sands in hollow cylinder torsional device. $\mathrm{PhD}$ thesis, Department of Civil Engineering, University of British Columbia, Vancour, BC (in preparation).

Shibuya, S. (1985). Undrained behaviour of granular materials under principal stress rotation. $\mathrm{PhD}$ thesis, University of London. 